\title{
Hipotireoidismo subclínico em idosos na atenção primária: ênfase na prevenção quaternária
}

\author{
Carla M. F. P. Neves, ${ }^{1 *}$ Michael Deveza, ${ }^{1}$ Rosimere J. Teixeira ${ }^{1}$
}

\begin{abstract}
Resumo
O diagnóstico do hipotireoidismo subclínico (HSC) no idoso tem elevado grau de dificuldade devido às mudanças decorrentes do envelhecimento. Nosso objetivo foi rastrear e avaliar o valor do TSH para intervenção do HSC em idosos assintomáticos acompanhados na Atenção Primária. Foram realizadas dosagens de T4l e TSH, utilizando as referências de TSH para adultos e faixa etária (grupos A: 60-69 anos; B: 70-79 anos e C: maior que 80 anos). O HSC foi definido como T4l normal e TSH acima limite normal até $10 \mathrm{mlU} / \mathrm{L}$. Foram avaliados 123 idosos, 35 homens e 88 mulheres. O HSC foi diagnosticado em $8,1 \%$ ( $n=10$ ) dos idosos, sem diferença por gênero. A média do TSH foi maior no grupo com mais de 80 anos ( $p=0,004)$. Foi observado aumento da proporção de HSC considerando o limite para adulto (A: 3,5\%; B: 5\% e C:15,4\%; p < 0,05) e faixa etária (A: 7\%; B: 5\% e C: 11,5\%; NS). Houve uma relação diretamente proporcional do TSH $\left(\mathrm{r}^{\wedge} 2=0,93, \mathrm{p}<0,01\right)$ e T41 $\left(\mathrm{r}^{\wedge} 2=\right.$ 0,54, p < 0,01) com a idade. Concluímos que o HSC é comum em idosos. Os níveis de TSH se correlacionam e aumentam com a idade, podendo significar um aumento fisiológico com possível papel cardioprotetor, melhor funcionalidade e maior longevidade. O TSH deve ser avaliado pela faixa etária e confirmado, evitando diagnósticos equivocados e tratamentos desnecessários, seguindo o preceito da prevenção quaternária. É importante que os médicos de família e comunidade, responsáveis pela coordenação do cuidado, estejam atentos à abordagem do HSC no idoso.
\end{abstract}

Descritores: Hipotireoidismo subclínico; Rastreamento; Idoso; Prevenção quaternária.

\section{Abstract \\ Subclinical hypothyroidism in elderly in primary care: emphasis on quaternary attention}

The diagnosis of subclinical hypothyroidism ( $\mathrm{SCH}$ ) in the elderly is highly difficult due to the changes of aging. Our goal was to track and assess the value of TSH for HSC intervention in asymptomatic elderly seen in primary care. We perform FT4 and TSH, TSH using the references for adults and age group (group A: 60-69 years; B: 70-79 years and C: greater than 80 years). The HSC was defined as normal FT4 and TSH above normal limit to $10 \mathrm{mIU} / \mathrm{L}$. We evaluated 123 elderly people, 35 men and 88 women. The HSC was diagnosed in $8.1 \%(n=10)$ of the elderly, with no difference by gender. The mean TSH was higher in the group with more than 80 years $(\mathrm{p}=0.004)$. We observed an increase of HSC ratio considering the limit for adult (A: 3.5\%; B: 5\% e C: 15.4\%; p < 0.05) and age group (A:

\section{Departamento de Medicina Integral, Familiar e Comunidade. Faculdade de Ciências Médicas. Universidade do Estado do Rio de Janeiro. Rio de Janeiro, RJ, Brasil.}

\author{
*Endereço para correspondência: \\ Rua Dois de Dezembro, 131, ap. 502 \\ Rio de Janeiro, RJ. CEP: 22220-040. \\ E-mail: carlamutto@globo.com
}

Revista HUPE, Rio de Janeiro, 2016;15(3):227-234

doi: 10.12957/rhupe.2016.29448

Recebido em 01/07/2016. Aprovado em 18/10/2016.

7\%; B: 5\% e C: $11.5 \%$; NS). There was a directly proportional relationship of TSH $\left(\mathrm{r}^{\wedge} 2=0.93, \mathrm{p}<0.01\right)$ and FT4 $\left(\mathrm{r}^{\wedge} 2=0.54\right.$, $\mathrm{p}<0.01)$ with age. We conclude that the HSC is common in the elderly. TSH levels correlate and increases with age, and can represent a physiological increase with a possible cardioprotective role, greater functionality and longevity. The TSH should be assessed by age and confirmed track, avoiding misdiagnosis and unnecessary treatments, following the precept of quaternary prevention. It is important that family and community doctors, responsible for the coordination of care, be attentive to the HSC approach in the elderly.

Keywords: Subclinical hypothyroidism; Tracking; Elderly; Quaternary prevention.

\section{Resumen}

Hipotiroidismo subclínico en mayores con atención primaria: énfasis en la atención cuaternaria

El diagnóstico de hipotiroidismo subclínico (SCH) en el anciano tiene un gran inconveniente debido a los cambios del envejecimiento. Nuestro objetivo fue realizar un seguimiento y evaluar el valor de TSH para la intervención HSC en ancianos asintomáticos tratados en atención primaria. Realizamos T4 libre y TSH - usando las referencias para adultos y edad (grupo A: 60-69 años; B: 70-79 años y C: mayor de 80 años). El HSC se definió como T4 libre y TSH normal, por encima del límite normal a $10 \mathrm{mUI} / \mathrm{L}$. Se evaluó a 123 ancianos, 35 hombres y 88 mujeres. El HSC se diagnosticó en el 8,1\% ( $n=10)$, sin diferencias por sexo. La media de TSH fue mayor en el grupo con más de 80 años ( $\mathrm{p}=0,004)$. Hemos observado un aumento de la proporción de HSC teniendo en cuenta el límite para un adulto (A: 3,5\%; B: 5\%; C: 15,4\%; p < 0,05) y edad (A: 7\%; B: 5\% e 


\section{Artigo original}

C: 11,5\%; NS). Hubo una relación directamente proporcional de TSH ( $\left.\mathrm{r}^{\wedge} 2=0,93, \mathrm{p}<0,01\right)$ y T4 libre $\left(\mathrm{r}^{\wedge} 2=0,54, \mathrm{p}<0,01\right)$ con la edad. Se concluye que la HSC es común en los ancianos. Los niveles de TSH se correlacionan y aumentan con la edad, y pueden significar un aumento fisiológico con un posible papel cardioprotector, mayor funcionalidad y longevidad. La TSH debe evaluarse por la edad y confirmarse la pista, evitando

\section{Introdução}

A prevenção quaternária foi definida como um conjunto de ações que se desenvolvem visando à identificação de pessoas em risco de iatrogenias médicas como excessos de intervenção e medicalização, com intuito de protegê-las de intervenções médicas inapropriadas e de lhes sugerir alternativas eticamente aceitáveis. ${ }^{1,2}$ Nesse contexto, entendemos que os idosos são os que mais sofrem com o intervencionismo diagnóstico e terapêutico, visto que a probabilidade de doenças aumenta. No entanto, é com base neste esse excesso de zelo que identificamos os idosos como um grupo favorecido com a aplicação da prevenção quaternária, reforçando então a necessidade de medidas preventivas como a educação em saúde e a constante atualização dos conhecimentos médicos.

O processo de envelhecimento está relacionado a alterações em vários níveis do eixo hipotálamo-hipófise-tireoide. A elevação do hormônio estimulador da tireoide (TSH) e o aumento dos títulos de autoanticorpos são as principais modificações encontradas. ${ }^{3}$

O hipotireoidismo subclínico (HSC) tem sido definido por níveis normais dos hormônios tireoidianos e elevados do TSH. ${ }^{4}$ Seu diagnóstico é estritamente laboratorial, pois os pacientes são assintomáticos, oligoassintomáticos ou com manifestações inespecíficas. Deve-se suspeitar e investigar os idosos que apresentem sintomas clínicos e/ou fatores de risco para hipotireoidismo como: mulheres acima de 60 anos; história pessoal ou familiar de doença tireoidiana, tireoidectomia, terapia com iodo radioativo ou radiação externa de pescoço; doença autoimune, em uso de medicamentos (lítio, amiodarona, interferon-alfa); doenças neuropsiquiátricas (depressão e demência), hiperprolactinemia, dislipidemia, anemia e insuficiência cardíaca (IC) (nível de recomendação D). ${ }^{5}$

O diagnóstico de HSC tem se tornado comum na prática ambulatorial devido ao maior acesso das pessoas e maior facilidade de solicitação de exames. Acomete $4-10 \%$ da população geral, mas sua prevalência aumenta com a idade, em mulheres com mais de 60 anos, doença autoimune e história familiar un mal diagnóstico y tratamientos innecesarios, siguiendo el precepto de la prevención cuaternaria. Es importante que los médicos de familia y la comunidad estén atentos a la aproximación de HSC en ancianos.

Palabras clave: Hipotiroidismo subclínico; Seguimiento; Ancianos; Prevención cuaternaria.

positiva. O envelhecimento influencia diretamente na sua prevalência. Estudos da função tireoidiana na população idosa descrevem uma prevalência entre 1-10\% de hipotireoidismo clínico (HC) e entre 1-15\% de HSC. ${ }^{6} \mathrm{O}$ HSC pode evoluir para o HC a uma taxa de $5 \%$ ao ano, especialmente na presença de anticorpos antitireoidianos. ${ }^{7,8}$ Estudos mostram que o HSC tem associação com os níveis aumentados de colesterol total e LDL, desta forma podendo agravar e/ou desenvolver doenças cardiovasculares. ${ }^{9}$

Existem muitas controvérsias em relação às recomendações para rastreio, valor de referência de TSH no diagnóstico e indicação do inicio do tratamento do HSC na população idosa. Além disso, seu início insidioso e os sintomas inespecíficos - queda de cabelos, pele seca, fraqueza, fadiga, intolerância ao frio e depressão - são frequentemente vistos como alterações do envelhecimento. $9,10,11$

O impacto que o hipotireoidismo pode causar na população idosa traz um desafio para o sistema público de saúde: a garantia de acompanhamento dos indivíduos identificados e o desenvolvimento de ações referentes à promoção da saúde e à prevenção de agravos. Este desafio é, sobretudo, da Atenção Básica, notadamente da Saúde da Família, espaço prioritário e privilegiado que atua com equipe multiprofissional e cujo processo de trabalho pressupõe vínculo com a comunidade, a clientela adscrita (número de pacientes por equipe), levando em conta diversidade racial, cultural, religiosa e os fatores sociais envolvidos. ${ }^{12}$

Para que a atenção integral aos idosos se efetive, devem-se programar estratégias diferenciadas que visem à participação comprometida dos indivíduos e seus familiares, promovendo autonomia destes. Devese também acolher a demanda da Atenção Primária à Saúde (APS) com mais cuidado, ou seja, tentar garantir o atendimento do idoso apesar da grande pressão das agendas e muitas vezes também da inadequação da clientela adscrita.

Como o diagnóstico do HSC no idoso tem elevado grau de dificuldade, as manifestações clínicas 
são confundidas comumente como sinais do próprio envelhecimento e existe uma alta morbimortalidade nesta população, nosso objetivo é rastrear a disfunção tireoidiana e avaliar o ponto de corte do TSH no diagnóstico do HSC em idosos acompanhados em uma Clínica de Família.

\section{Metodologia}

Trata-se de um estudo de corte transversal, realizado durante a residência médica na Clínica da Família Ana Maria da Conceição dos Santos Correia, na Equipe Servino Mengarda, no bairro Vicente de Carvalho, na cidade do Rio de Janeiro.

Foram avaliados idosos, ou seja, indivíduos com idade igual ou superior a 60 anos de ambos os sexos. ${ }^{13}$ Os pacientes foram divididos em três grupos de acordo com a faixa etária (A: 60-69 anos; B: 70-79 anos e C: maior que 80 anos).

A disfunção tireoidiana foi rastreada em 142 idosos por meio da busca ativa das dosagens séricas de TSH e T4l contidas nos prontuários de indivíduos sem sinais e/ou sintomas de hipotireoidismo ou oligossintomáticos. Posteriormente à busca ativa desses resultados foi realizada consulta para confirmação dos dados clínicos e exclusão dos casos com doença tireoidiana prévia.

O HSC foi definido pelos níveis de T4l normal e TSH acima do limite normal até $10 \mathrm{mlU} / \mathrm{L}$. O HC foi considerado na presença de TSH maior que $10 \mathrm{mIU} / \mathrm{L}$. Os valores acima do normal para o T4l e menores que o normal para TSH caracterizaram o hipertireoidismo subclínico. ${ }^{2}$

Considerou-se como referência normal para adultos os valores de TSH entre 0,34 a 5,60 uIU/mL e de T4l entre 0,54 a 1,60 ng/dL, dosados pelo método de quimiofluorescência do laboratório AFIP Medicina Diagnóstica. Entretanto, o TSH também foi avaliado quanto ao limite de normalidade para faixa etária (A: TSH até 4,7 uIU/mL; B: TSH até 5,6 uIU/mL e C: TSH até $6,3 \mathrm{uIU} / \mathrm{mL}$ ), com base no Consenso sobre Doença Tireoidiana da Sociedade Brasileira de Endocrinologia e Metabologia., ${ }^{2,14}$

$\mathrm{Na}$ análise dos resultados do TSH, os pacientes foram classificados como normais para limite do laboratório; normais para o limite da idade; e presença de HSC ou HC, como descrito acima.

Foram coletados no prontuário de cada paciente e arquivadas no sistema informático da clínica de família (Vita Care) os seguintes dados: sexo, idade, diagnóstico prévio de hipotireoidismo, uso de medicamentos para disfunção da glândula e/ou que interfiram na função tireoidiana como amiodarona, corticoide, interferon, entre outros, além das principais comorbidades do paciente.

O tratamento estatístico das variáveis estudadas foi feito no programa Epi-Info versão 3.4. Para a análise das variáveis numéricas utilizamos os testes de ANOVA e Krwskal-Walls e para as variáveis categóricas utilizamos o teste Qui-quadrado ou teste exato de Fisher, considerando significativo o valor do $p<0,05$. A associação entre o valor do TSH e do T4 livre foi determinada pelo coeficiente de correlação de Pearson.

\section{Resultados}

Foram selecionados 123 idosos, com idades entre 60 e 93 anos (mediana de 71), sendo 35 (28,5\%) do sexo masculino e 88 (71,5\%) do feminino. Os idosos foram classificados por faixa etária como grupo A, 57 pacientes com 60,2 $\pm 2,3$ anos; grupo B, 40 pacientes com $74,5 \pm 2,9$ anos e grupo C, 26 pacientes com 82,8 \pm 2 ,1anos.

Do total de idosos avaliados, 108 (88\%) tinham função tireoidiana normal, dentro dos limites normais de referência para adultos. Nos idosos com alteração da função tireoidiana $(\mathrm{n}=15)$ o diagnóstico definitivo foi de: HC ( $n=3-2,4 \%)$ e HSC ( $n=10-8,1 \%)$ e hipertireoidismo subclínico (Hiper: $\mathrm{n}=2-1,6 \%$ ). Após a busca ativa dos resultados do TSH e avaliação clínica foram excluídos 19 pacientes com hipotireoidismo prévio, sendo que 8 apresentavam níveis hormonais normais e o restante mantinha-se descompensado 9 com níveis de TSH elevado e 2 com níveis suprimidos).

Ao analisarmos o total de diagnósticos não observamos diferença por sexo, sendo 10 mulheres $(11,4 \%$ - 3 HC, 6 HSC 1 Hiper) 5 homens (14,3\% - 4 HSC e 1 Hiper).

Quando analisamos o número de casos de $\mathrm{HC}$ e HSC, observamos um aumento da proporção de casos de hipotireoidismo no grupo mais idoso, sendo: 4 HSC no grupo A ( $7 \%$ - $n=57), 2$ HSC e 1 HC no B ( $5 \%$ e $2,5 \%$, $\mathrm{n}=40)$ e 4 HSC e 2 HC no C $(15,4 \%$ e $7,7 \%, \mathrm{n}=26)(\mathrm{p}=$ 0,07 ) (Tabela 1).

Ao analisar o TSH, considerando o valor de referência para adulto, observamos que o TSH encontrava-se no limite normal em $87,8 \%(\mathrm{n}=108)$ e elevados em $12,2 \%(\mathrm{n}=15)$. Já quando analisamos o TSH por faixa etária, $81,3 \%(\mathrm{n}=100)$ apresentavam níveis normais e $18,7 \%(n=23)$ acima da normalidade.

A média do TSH nos 123 idosos selecionados foi de $4,2 \pm 1,2 \mu \mathrm{U} / \mathrm{mL}$ (mediana de 2,2 ) e do T4 livre de 1,2 $\pm 0,9 \mathrm{mg} / \mathrm{dL}$ (mediana de 0,93 ). Analisando o nível do TSH por grupo, a média foi de $3,4 \pm 3,8(0,1$ a 57,4$) \mu \mathrm{U} /$ $\mathrm{mL}$ no $\mathrm{A} ; \mathrm{de} 2,9 \pm 3,8(0,02$ a 38,7$) \mu \mathrm{U} / \mathrm{mL}$ no B e de $7,7 \pm 14$ 


\section{Artigo original}

$(0,6$ a 85,8$) \mu \mathrm{U} / \mathrm{mL}$ no C. Enquanto a média do T41 foi de 1,2 \pm 0,5 mg/dL; 0,96 \pm 0,4 mg/dL e 1,5 $\pm 1,7 \mathrm{mg} / \mathrm{dL}$ no grupo A, B e C, respectivamente. Os níveis de TSH foram maiores no grupo $C(p=0,004)$ (Figura 1), mas sem diferença quanto ao T4 livre.

Quando analisamos a proporção de casos de HSC, considerando o TSH para o limite normal de adulto, observamos um aumento desta proporção por faixa etária ( $\mathrm{p}<0,05)$, sendo $2 / 57$ no grupo A $(3,5 \%)$; 2/40 no B (5\%) e 4/26 no C (15,4\%) (Figura 2).

Entretanto, quando analisamos a proporção de HSC, considerando o TSH para a normalidade por faixa etária, notamos uma maior proporção de casos no grupo C (3/26-11,5\%) do que no A (4/57-7\%) e no B $(2 / 40-5 \%)(p=$ NS) (Figura 2).

$\mathrm{Na}$ análise de regressão linear, observou-se uma relação diretamente proporcional dos níveis de TSH $\left(\mathrm{r}^{\wedge} 2=0,93, \mathrm{p}<0,01\right)$ e de T4l $\left(\mathrm{r}^{\wedge} 2=0,54, \mathrm{p}<0,01\right)$ com a idade (Figura 3).

\section{Discussão}

De acordo com dados de 2013 do IBGE (Instituto Brasileiro de Geografia e Estatística), a expectativa de vida dos brasileiros está beirando os 74 anos; e as pessoas com mais de 60 anos já representavam 12,6\% da população, em 2011, sendo a maior parte mulheres. ${ }^{15}$

Com o envelhecimento da população, estamos diante de um novo desafio, abordar doenças que antes não eram comuns nessa faixa etária, como o HSC. No Brasil, a prevalência de TSH elevado em uma amostra de 1.220 mulheres adultas da cidade do Rio de Janeiro foi de 12,3\%, alcançando 19,1\% entre aqueles acima de

Tabela 1. Distribuição dos idosos por faixa etária de acordo com a presença ou não de doenças da tireoide

\begin{tabular}{|c|c|c|c|c|}
\hline Faixa etária & $\begin{array}{c}\text { Eutireoidismo } \\
\text { NO-\% }\end{array}$ & $\begin{array}{c}\text { HC } \\
\text { NO-\% }\end{array}$ & $\begin{array}{l}\text { HSC } \\
\text { NO-\% }\end{array}$ & $\begin{array}{c}\text { Hiper SC } \\
\text { N0-\% }\end{array}$ \\
\hline $\begin{array}{c}\text { A: } 60-69 \text { anos } \\
\text { Total: } 57\end{array}$ & $\begin{array}{c}52 \\
91,2 \%\end{array}$ & 0 & $\begin{array}{c}4 \\
7 \%\end{array}$ & $\begin{array}{c}1 \\
1,8 \%\end{array}$ \\
\hline $\begin{array}{c}\text { B: } 70-79 \text { anos } \\
\text { Total: } 40\end{array}$ & 36 & $\begin{array}{c}1 \\
2,5 \%\end{array}$ & $\begin{array}{c}2 \\
5 \%\end{array}$ & $\begin{array}{c}1 \\
2,5 \%\end{array}$ \\
\hline $\begin{array}{c}\text { C: } \geq 80 \text { anos } \\
\text { Total: } 26\end{array}$ & $90 \%$ & $\begin{array}{c}2 \\
7,7 \%\end{array}$ & $\begin{array}{c}4 \\
15,4 \%\end{array}$ & 0 \\
\hline
\end{tabular}

$p=0,07$

Eutireoidismo = função tireoidiana normal; HC = hipotireoidismo clínico; HSC = hipotiroidismo subclínico e HiperSC = hipertireoidismo subclínico.

Figura 1. Nível médio de TSH entre as faixas etárias

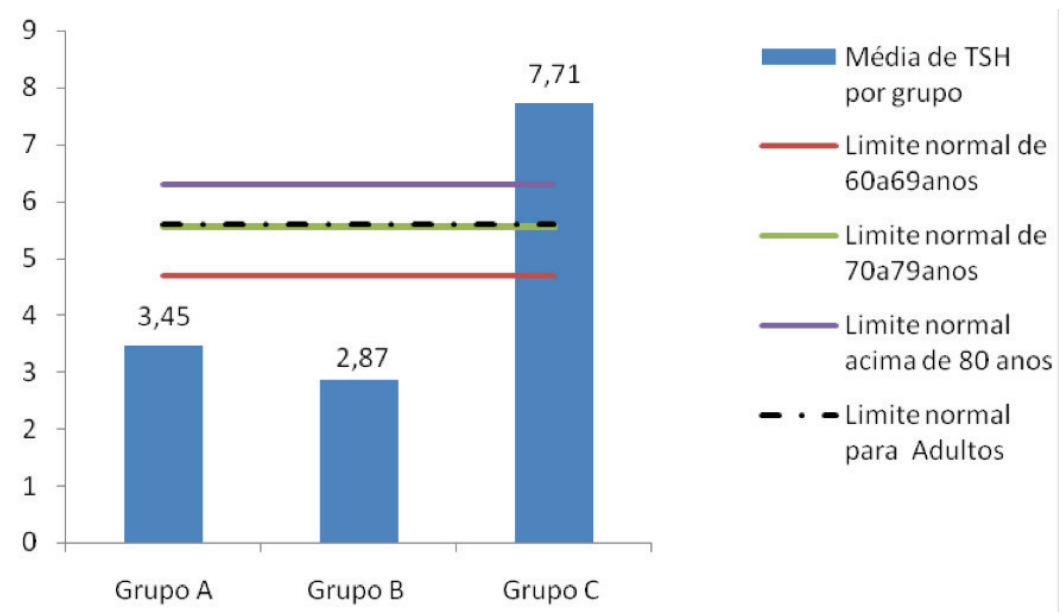

Na figura 1: o nível médio do TSH foi significativamente diferente entre os grupos por faixa etária $(p=0,004)$. As linhas contínuas representam o limite normal para as faixas etárias e a linha tracejada preta representa o limite normal para adultos. 
70 anos. ${ }^{16}$ Na população idosa da cidade de São Paulo, a prevalência de HSC foi de 6,5\% e 6,1\%, em mulheres e homens, respectivamente. ${ }^{17}$ No mesmo estudo encontramos 8,1\% de HSC nos idosos, também sem diferença na proporção de disfunção tireoidiana entre os gêneros. Entretanto, esses autores não avaliaram o ponto de corte do TSH por faixa etária como no nosso estudo.

Um número crescente de estudos sobre o hipotireoidismo no idoso tem sido desenvolvido, pelo fato de que o TSH pode ser fisiologicamente elevado. ${ }^{9}$ Como essas alterações ainda não são totalmente compreendidas, resultam em controvérsias quanto ao seu rastreio, diagnóstico e tratamento.

Os níveis de referência para o TSH na população geral são obtidos pela média de uma população saudável supostamente sem doenças tireoidianas. ${ }^{18}$ Entretanto, obter tal tipo de população é difícil e os valores de referência comumente utilizados para as

Figura 2. Proporção de idosos com níveis de TSH elevados para o limite normal de adulto e para o limite normal para a faixa etária

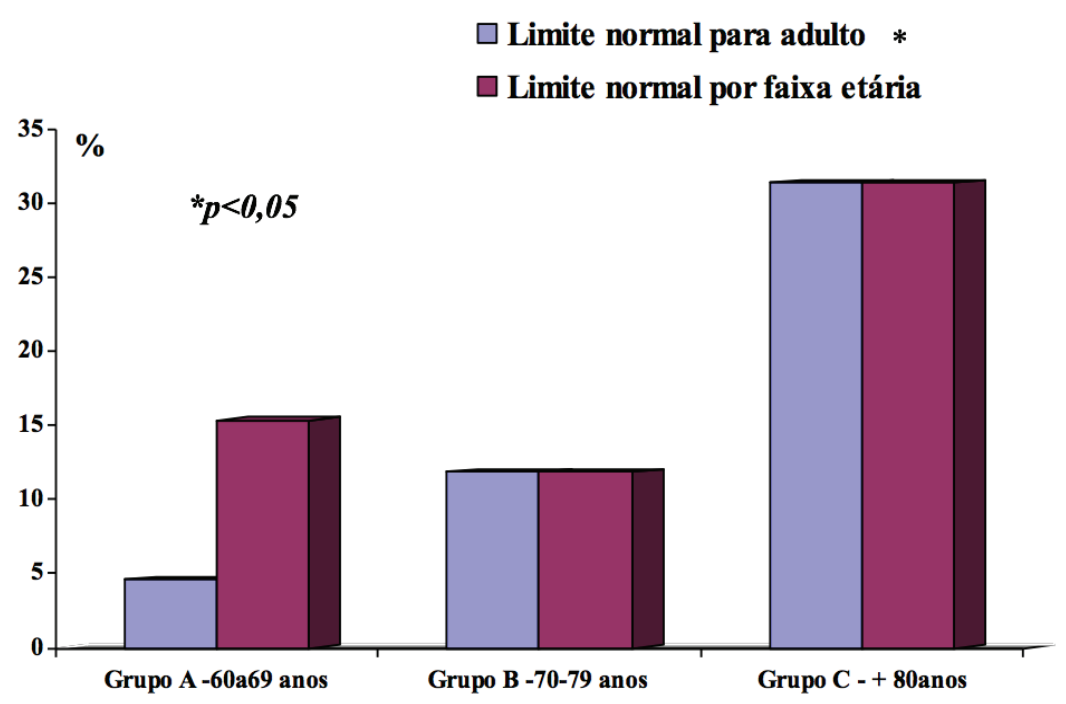

Figura 3. Regressão linear entre os níveis de TSH e a idade em anos

Associação entre os níve is de TSH e a idade

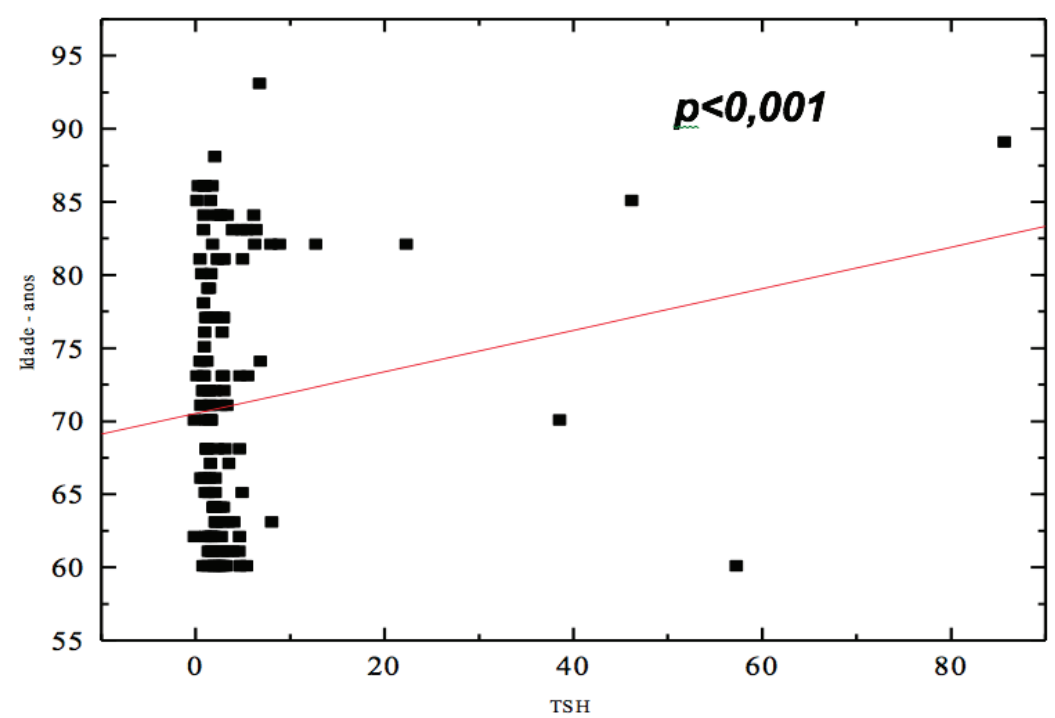




\section{Artigo original}

raças, gêneros e etnias provêm, atualmente de grandes estudos norte-americanos, que definem como valores de referência para adultos normais os níveis séricos do TSH entre 0,4 e 4,5 $\mu \mathrm{U} / \mathrm{L}$ (nível de recomendação A). ${ }^{14,19}$ Contudo, deve-se lembrar do efeito da idade no limite superior do que se deve considerar como normal. ${ }^{14}$

Um estudo brasileiro analisou o TSH em 13.296 indivíduos de diferentes idades sem doença tireoidiana aparente, demonstrando aumento dos níveis médios de TSH nos mais idosos. ${ }^{2}$ Em nosso estudo, também observamos a elevação dos níveis médios de TSH com a idade. A proporção de casos com HSC, analisando o valor de referência normal para adultos, foi significativamente maior nos mais idosos. No entanto, quando avaliamos os valores de TSH para a normalidade por faixa etária, percebemos apenas uma maior proporção de HSC naqueles com mais de 80 anos. Como o limite normal de referência do laboratório que utilizamos era maior do que os utilizados pela literatura, isso pode ter interferido nos resultados especialmente do grupo entre 60-69 anos. Uma limitação de nosso estudo foi que a situação da tireoide baseou-se em um único teste, logo, a reprodutibilidade não foi avaliada. É possível que alguns pacientes com TSH em torno do ponto de corte tenham sido classificados erroneamente como HSC e representem apenas uma elevação fisiológica ou transitória do TSH.

Esses dados enfatizam a importância de se definir um ponto de corte para o TSH em idosos. No Consenso Brasileiro para a abordagem do HSC foi recomendado utilizar o valor de referência de acordo com os intervalos de normalidade por faixa etária (nível de recomendação B). ${ }^{2,20}$ Como ainda não há um valor definido para o TSH, um excessivo número de diagnósticos de HSC pode ocorrer nessa população.

Quanto ao rastreio do hipotireoidismo, recomendase para todos os pacientes acima de 60 anos (nível de recomendação B), devido à elevada prevalência nesta faixa etária. As dosagens de TSH e T4l devem ser solicitadas na suspeita clínica de hipotireoidismo. Nos demais grupos de risco, recomenda-se dosar o TSH e, se entre 4,5$10 \mu \mathrm{U} / \mathrm{L}$, deve ser repetido após 2-3 meses e complementar com o T4 livre e anti-TPO (nível de recomendação D). ${ }^{2,21}$ De todo modo, o posicionamento da Força-Tarefa de Serviços Preventivos dos Estados Unidos que está em processo de atualização, mas ainda não concluído, mostra que as evidências atuais são insuficientes para avaliar os benefícios e prejuízos em rastrear disfunção tireoidiana em adultos assintomáticos. ${ }^{22}$

Uma proporção significativa de pacientes com HSC apresenta normalização do TSH durante os 2 a 5 anos de seguimento, em especial aqueles com TSH $\pm \leq 10$ $\mu \mathrm{U} / \mathrm{L} .{ }^{23}$ Os níveis de TSH $\pm \geq 10 \mu \mathrm{U} / \mathrm{L}$ associam-se com maior risco de progressão ao $\mathrm{HC}^{24}$ de doença arterial coronariana (DAC) e de morte. ${ }^{25}$ Então, o HSC poderia ser classificado, de acordo com o TSH, em leve-moderado (TSH 4,5-9,9 $9 \mathrm{U} / \mathrm{L}$ ) e grave $(\mathrm{TSH} \pm 10 \mu \mathrm{U} / \mathrm{L}$ ) (nível de recomendação D). ${ }^{26}$

Os efeitos do HC sobre a qualidade de vida são bem estabelecidos, mas permanecem controversos no HSC. Isso é preocupante, pois muitas vezes inicia-se o tratamento do HSC com base nas evidências consistentes sobre associação de HSC com risco de DAC. Porém, não há confirmação dessa relação em idosos maiores de 65 anos (nível de recomendação A). ${ }^{27}$ Além disso, acreditase que a elevação fisiológica de TSH (entre 4,5 a 10 uIU/ $\mathrm{mL}$ ) pode representar um fator de proteção cardiovascular, melhor funcionalidade e maior longevidade em idosos com mais de 85 anos. ${ }^{7,20,27}$

O tratamento do hipotireoidismo deve ser individualizado e deve buscar preservar a qualidade de vida do idoso. Naqueles com mais de 65 anos, o HSC deve ser tratado somente quando o TSH estiver persistentemente igual ou superior a $10 \mathrm{uIU} / \mathrm{mL}$ (nível de recomendação D). 2,3,28 Também deve ser considerado no HSC grave com anti-TPO positivo e alterações ultrassonográficas sugestivas de tireoidite, pois apresentam risco de evolução para HC, IC e mortalidade cardiovascular. 3,22,25,28 Porém, há situações com recomendações terapêuticas diferentes no HSC leve-moderado., ${ }^{2,3}$

A dose de levotiroxina no idoso situa-se em torno de $1 \mathrm{mcg} / \mathrm{Kg} / \mathrm{dia}$, diferentemente dos adultos, que é de $1,7 \mathrm{mcg} / \mathrm{Kg} /$ dia. No entanto, recomenda-se que em idosos com DAC e IC, deva-se iniciar a terapia em doses mais baixas (12,5-25 mcg/dia), especialmente no HSC.,3 Os ajustes devem ser orientados pela resposta clínica e níveis do TSH. ${ }^{21,22}$ Os estudos nem sempre mostram evidências sustentadas de reversão do quadro clínico, e, caso esta seja a única indicação e a clínica permaneça inalterada após normalização do TSH, o tratamento deve ser suspenso. ${ }^{21}$

Neste estudo, chamou atenção o fato de que nos pacientes que foram excluídos por apresentar diagnóstico prévio, em mais na metade dos casos, o controle era inadequado. A falta de adesão ao tratamento, a polifarmácia e a presença de multimorbidades podem contribuir para o mau controle do hipotireoidismo no idoso. ${ }^{29}$ Cerca de $40 \%$ dos idosos em tratamento utilizam doses suprafisiológicas de levotiroxina, resultando em hipertireoidismo clinico ou subclínico. Esse fato 
adquire grande relevância nos idosos, pois está associado ao risco de fibrilação atrial e perda de massa óssea. ${ }^{29}$

Os níveis de TSH aumentam com a idade, podendo representar uma adaptação fisiológica com possível papel cardioprotetor, melhor funcionalidade e maior longevidade.,20,27 Acreditamos que o hipotireoidismo não deva ser rastreado em todos os idosos, apenas quando houver suspeita clínica ou em grupos de risco. ${ }^{3,22}$ Então, reforçamos a importância das recomendações para uma abordagem individualizada do idoso com hipotireoidismo. Devemos evitar procedimentos desnecessários, diagnóstico equivocado, medicalização excessiva e encaminhamentos precipitados ao endocrinologista, obedecendo às características do sistema de saúde brasileiro. Na presença de profissionais de saúde capacitados, devemos encaminhar somente em situações como doenças cardíacas, outras doenças endócrinas associadas e pacientes de difícil controle. ${ }^{21}$

Como a APS é responsável pelo cuidado das condições mais frequentes, torna-se imperativo que os médicos de família e comunidade, os coordenadores do cuidado, sejam capacitados a cuidar desses casos. A criação de programas para capacitar profissionais na abordagem do hipotireoismo, com recomendações específicas para o HSC, pode resultar na promoção da saúde, redução da morbimortalidade e prevenção de iatrogenias. Evitando assim os erros de intervencionismo diagnóstico e terapêutico, e garantindo a qualidade de vida do idoso. Além disso, para um resultado positivo no curso da doença, é necessário um bom vínculo entre profissional de saúde/equipe e pessoa/familiar/ cuidador para que haja diálogo e bom entendimento da patologia e terapêutica.

\section{Conclusão}

O HSC é comum em idosos. Os níveis de TSH se correlacionam e aumentam com a idade, podendo significar um aumento fisiológico com possível papel cardioprotetor, melhor funcionalidade e maior longevidade. Os valores de TSH devem ser avaliados de acordo com a faixa etária e sempre confirmados, evitando diagnósticos equivocados e tratamentos desnecessários, seguindo o preceito da prevenção quaternária.

\section{Referências}

1. Jamoulle $M$. Information et informatisation en médecine générale. In: Berleur J, Labet-Maris Cl, Poswick RF, Valenduc G, Van Bastelaer Ph. Les informa-g-iciens. Namur (Belgique): Presses Universitaires de Namur; 1986. p.193-209.

2. Jamoulle M. Quaternary prevention, an answer of family doctors to overmedicalization. International Journal of Health
Policy and Management. 2015;4(2):1-4.

3. Veldhuis JD. Changes in pituitary function with aging and implications for patients care. Nature Reviews Endocrinology, London. 2013;9(4):205-15.

4. Sgarbi JA, Teixeira PFS, Maciel LMZ, et al. Consenso brasileiro para a abordagem clínica e tratamento do hipotireoidismo subclínico em adultos: Recomendações do Departamento de Tireoide da Sociedade Brasileira de Endocrinologia e Metabologia. In: Arq Bras Endocrinol Metab. São Paulo. 2013;57(3):167-75.

5. Brenta G, Vaisman M, Sgarbi JA, et al. Diretrizes clínicas práticas para o manejo do hipotiroidismo. In: Arq Bras Endocrinol Metab. São Paulo. 2013;57(4):265-9.

6. Bensenor IM, OImos DR, Lotufo PA. Hypothyreoidism in the elderly: diagnosis and management. Clinical Interventions in Aging, Auckland. 2012;7:97-111.

7. Gharib H, Tuttle RM, Baskin HJ, et al. Consensus Statement: Subclinical thyroid dysfunction; A Joint statement on Management from the American Association of Clinical Endocrinologists, the American Thyroid Association, and the Endocrine Society. J Clin Endocrinal Metab. 2005;90(1):581-5.

8. Biondi B, Cooper D. The clinical significance of subclinical thyroid dysfunction. Endo Review. 2008;29(1):76-131.

9. Paz-Filho G, Graf H. Hipotireoidismo Subclínico in Programa de atualização em doenças da tireoide da SBEM; Fascículo 2; p. 4-7.

10. Vaisman M. Hipotireoidismo em Situações Especiais: Hipotireoidismo e o Idoso in Programa de atualização em doenças da tireoide da SBEM; 2009;3:4-5.

11. Boelaert K. Thyreoid disfunction in the elderly. Nature Reviews Endocrinology, London. 2013;9(4):194-204.

12. Cunha C. Atenção Básica de Saúde Através do Hiperdia: Plano de Reorganização da Atenção; p. 17. Porto Alegre 2009. Em: http://www.lume.ufrgs.br/bitstream/handle/10183/17937/000725363.pdf.

13. Brasil. Lei N 10.741, 1 de outubro de 2003 - Estabelece diretrizes sobre a política nacional do idoso (PNI).

14. Boucai L, Hollowell JG, Surks MI. An approach for development of age-, gender and ethnicity-specific thyrotropin reference limits. Thyroid. 2011;21(1):5-11.

15. Instituto Brasileiro de Geografia e Estatística. Pesquisa Nacional por Amostra de Domicílios. Rio de janeiro: IBGE; 2013.

16. Sichieri R, Baima J, Marante T, et al. Low prevalence of hypothyroidism among black and Mulatto people in a population-based study of Brazilian women. Clin Endocrinol. Oxford. 2007;66(6):803-7.

17. Benseñor IM, Goulart AC, Lotufo PA, et al. Prevalence of thyroid disorders among older people: results from the São Paulo Ageing \& Health Study. Cad Saude Publica. 2011;27(1):155-61.

18. Baloch Z, Carayon P, Conte-Devolx B, et al. Guidelines Committee, National Academy of Clinical Biochemistry. Laboratory Medicine Practice Guidelines: Laboratory Support for the Diagnosis and Monitoring of Thyroid Disease. Thyroid. 2003;13(1):57-67.

19. Hollowell JG, Staehling NW, Flanders WD, et al. Serum TSH, $\mathrm{T} 4$, and thyroid antibodies in the United States population (1988 to 1994): National Health and Nutrition Examination Survey (NHANES III). J Clin Endocrinol Metab. 2002;87(2):489-99.

20. Atzmon G, Barzilai N, Hollowell JG, et al. Extreme longevity is associated with increased serum thyrotropin. J Clin Endocrino Metab. 2009;94(4):1251-4.

21. Garber J, Cobin RH, Gharib H, et al. Clinical practice guidelines for hypothyroidism in adults: cosponsored by the Ameri- 


\section{Artigo original}

can Association of Clinical Endocrinologists and the American Thyroid Association. In: Thyroid; Revista Oficial da American Thyroid Association. 2013;2:27-33.

22. Draft Recommendation Statement: Thyroid Dysfunction: Screening U.S. Preventive Services Task Force. October 2014 Disponível em: http:/www.uspreventiveservicestaskforce.org/ Page/Document/RecommendationStatementDraft/thyroid-dysfunction-screening. Acesso em: 17 fev, 2015.

23. Meyerovitch J, Rotman-Pikielny P, Sherf M, et al. Serum thyrotropin measurements in the community: five-year follow-up in a large network of primary care physicians. Arch Intern Med. 2007;167(14):1533-8.

24. Diez JJ, Iglesias P. Spontaneous subclinical hypothyroidism in patients older than 55 years: an analysis of natural course and risk factors for the development of overt thyroid failure. J Clin Endocrinol Metab. 2004;89(10):4890-7.

25. Rodondi N, den Elzen WP, Bauer DC, et al. Subclinical hypo- thyroidism and the risk of coronary heart disease and mortality. Thyroid Studies Collaboration. JAMA. 2010;304(12):1365-74.

26. Cooper DS, Biondi B. Subclinical thyroid disease. Lancet. 2012;379 (11):1142-54.

27. Razvi S, Shakoor A, Vanderpump M, et al. The influence of age on the relationship between subclinical hypothyroidism and ischemic heart disease: a metaanalysis. J Clin Endocrinol Metab. 2008;93(8):2998-3007.

28. Fatourechi V, Aniszewski JP, Fatourechi GZ, et al. Clinical features and outcome of subacute thyroiditis in an incidence cohort: Olmsted County, Minnesota, study. J Clin Endocrinol Metab. 2003;88(5):2100-5.

29. Somwaru L.L, Arnold AM, Joshi N, et al. High frequency of and factors associated with thyroid hormone over-replacement and under-replacement in men and women aged 65 and over. The Journal of Clinical Endocrinology and metabolism. 2009;94(4):1342-5. 